\title{
Study Effect of Vitamins D3 and B6 Supplementation on Treatment Response in Breast Cancer Using Antibody-Coated Magnetic Nanoparticles Immunoassay for Circulating Endothelial Cells
}

\author{
Hewida Hassan Fadel ${ }^{1, ~ *, ~ M o h a m e d ~ A h m e d ~ A b d e l ~ M o h s e n ~}{ }^{2}$, Khaled El Sayed Soliman ${ }^{3}$, \\ Hanaa Mohamed Kohail ${ }^{4}$, Shehata Mahmoud EL Sewedy ${ }^{1,2}$ \\ ${ }^{1}$ Medical Laboratory Technology Department/ Faculty of Allied Medical Science, Pharos University in Alexandria, Alexandria, Egypt \\ ${ }^{2}$ Applied Medical Chemistry Department/ Medical Research Institute, University of Alexandria, Alexandria, Egypt \\ ${ }^{3}$ Experimental and Clinical Surgery Department/ Hospital of Medical Research Institute, University of Alexandria, Alexandria, Egypt \\ ${ }^{4}$ Cancer Management and Research Department/ Hospital of Medical Research Institute, University of Alexandria, Alexandria, Egypt

\section{Email address:} \\ hewida.fadel@pua.edu.eg (H. H. Fadel), prof_abdelmohsenma@yahoo.com (M. A. A. Mohsen), \\ khaled10363@yahoo.com (K. El S. Soliman), hmkohail@hotmail.com (H. M. Kohail), shehata.elsewedy@pua.edu.eg (S. M. EL Sewedy) \\ ${ }^{*}$ Corresponding author
}

\section{To cite this article:}

Hewida Hassan Fadel, Mohamed Ahmed Abdel Mohsen, Khaled El Sayed Soliman, Hanaa Mohamed Kohail, Shehata Mahmoud EL Sewedy. Study Effect of Vitamins D3 and B6 Supplementation on Treatment Response in Breast Cancer Using Antibody-Coated Magnetic Nanoparticles Immunoassay for Circulating Endothelial Cells. Cancer Research Journal. Vol. 6, No. 3, 2018, pp. 92-100. doi: $10.11648 /$ j.crj.20180603.14

Received: June 26, 2018; Accepted: July 9, 2018; Published: August 2, 2018

\begin{abstract}
In cancer patients, oxidative stress (OS) plays an important role in the initiation, promotion, progression of disease, in addition to, the continuous exposure to OS throughout the surgical procedure, chemotherapy and radiotherapy. The present study was designated to evaluate the role of vitamin D3 and B6 supplementation in combination with anti-cancer therapies in maintenance the redox status and their impact on angiogenesis, the main step in cancer progression and aggressiveness, as well as on the side effect of surgery, chemotherapy and/or radiotherapy. Our investigations were at two levels, firstly, assessment of chemical parameters for redox status and angiogenic factors, secondly, assessment level of circulating endothelial cells by applying immunoassay using antibodies coupling nanoparticles. Blood samples were collected once from 15 apparently healthy women (GP-I) and four times from 20 BC patients; (GpII)before mastectomy (B. M.), after mastectomy (A. M.), after ending of chemotherapy course (A. C.) and after 3 months later (A.3M) for both subgroups to Monitor the oxidant-antioxidants status (MDA and TAS) and angiogenic biomarkers (VEGF, ES and CECs). Our data reveals that comparing to control group, BC patients show significant elevation of MDA, VEGF and CECs $(P<0.001)$ as well as ES $(P<0.05)$. The serum level of CECs decreased significantly $(P<0.001)$ in $\mathrm{BC}$ patients supplemented with vitamins from the beginning of chemotherapy comparing to those receiving chemotherapy alone and then supplemented with vitamins for three months later. Supplementation with vitamins D3 and B6 reduces the oxidative stress, the side effects of chemotherapy and radiotherapy as vomiting and fatigue, in addition to interfere with angiogenesis.
\end{abstract}

Keywords: Breast Cancer, Vitamins D3 and B6, Angiogenesis, VEGF, ES, Circulating Endothelial Cells

\section{Introduction}

Breast cancer (BC) is the greatest lethal cancer among women worldwide. In Egypt, BC incidence is estimated for
$37.7 \%$ of all reported tumor and high mortality rate in addition to the aggressiveness of disease due to limited access of early diagnosis in rural areas [1]. Several risk factors increase the incidence of $\mathrm{BC}$ as age, reproductive and menstrual history, hormonal factors, family history, lifestyle, 
environmental factors and race [2]. Of these, OS plays the major role from the initiation to promotion of carcinogenesis and throughout the treatment strategies including surgery, chemotherapy and radiotherapy. Additionally, continuous generation of ROS within mammalian cells enhances the tumor cell production of the angiogenic factors which lead to increase risk factor of cell motility, invasion, metastasis, recurrence and aggressiveness of BC. Therefore, exacerbation of OS consequences can be contributed to the failure in antioxidant defense mechanisms in cancer patients $[3,4]$. Tumor cells secrete a large amount of pro-angiogenic factors, of these; Vascular Endothelial Growth Factor (VEGF) which plays the major role. On other hand, the main anti-angiogenic factor ES induced tumor regressions by inhibiting of ECs proliferation and increasing tumor cell apoptosis. Subsequently, losing of angiogenic balance in cancer cells directed several studies to focus on inhibition of angiogenesis that is the most important factor in the progression of cancer as well as the most promising novel approaches for the treatment of cancer [5].

In addition, enumeration and characterization of CECs may offer a unique opportunity for monitoring the efficacy of and the optimal biological dose of anti-angiogenesis therapies [6]. Vitamin D3 involves in cell cycle regulation and reduces both incidence and recurrence rate of $\mathrm{BC}$ through a variety of different mechanisms as it induces BRCA1 gene expression, differentiation, apoptosis, inhibition of cellular proliferation and angiogenesis in normal and malignant breast cell [7]. Similarly, vitamin B6 serves as a coenzyme for many reactions that suppress cell proliferation, angiogenesis and subsequently reduce risk of cancer $[8,9]$. The present study intends to clarify the major issues surrounding the controversy of vitamin supplementation in combination with standard therapy for $\mathrm{BC}$ by assessment of oxidative homeostasis and angiogenic balance markers to understand whether there are benefits of vitamins D3 and B6 supplementation, also to clarify the interaction between vitamins and conventional anti-cancer therapy.

\section{Materials and Methods}

\subsection{Subjects}

Informed consent was obtained from all participants, and the study was approved by the research Ethics board of Medical Research Institute, Alexandria University. The present study is carried out on 15 healthy women as control group (Gp-I) and $20 \mathrm{BC}$ patients who have been histopathologically proved BC (Gp-II). All patients were diagnosed with primary infiltrating ductal cancer of breast (stage II, III) ( age: 45 \pm 1.7 ) who had underwent modified radical mastectomy at Cancer Management and Research Department and the Clinical Surgical department of Medical Research Institute (MRI). Patients who were pregnant, lactating women, aged more than 70 years old were excluded. Only BC patients that did not receive pre-operative neoadjuvant chemotherapy were enrolled in our study.

After mastectomy, Gp-II was divided into two subgroups; 1) Gp-IIa; 10 patients received the recommended chemotherapy and 2) Gp-IIb; 10 patients received recommended chemotherapy plus supplementation with D3 (800 IU/day) and vitamin B6 (50mg/day) $[6,8]$. At the end of chemotherapy cycles, the patients of both subgroups (Gp-IIa and $\mathrm{Gp}$-IIb) were supplemented with the same doses of vitamin B6 and D3 for three months.

Blood Samples were collected from the 20 patients with BC one day before mastectomy (B. M.), 2 days after mastectomy (A. M.), at the end of chemotherapy cycles (A. C.) and finally after three months later $(\mathrm{A} .3 \mathrm{M})$. Blood Samples from 15 healthy women as control group (Gp-I) were also collected. Samples were divided as following: 1) Fresh whole blood was prepared for determination of CECs; 2) Serum was prepared and divided into aliquots and frozen at $-80^{\circ} \mathrm{C}$ for the biochemical investigations of MDA, TAS, VEGF and ES.

\subsection{Chemicals \& Kits}

All the chemicals used in the study were of analytical grade and purchased from Sigma-Aldrich (St. Louis, MO, USA). Serum level of VEGF and ES were measured using ELISA Kits from (Bender MedSystems GmbH, Vienna and Ray Biotech, Inc.) respectively) according to manufacture instructions. Assessment of CECs in peripheral blood was carried out by using Dynabeads M-450 Pan Mouse IgG (DYNAL Biotech) which were coated with murine anti-CD146 antibody (BioCytex Marseille, France).

\subsection{Biochemical Investigations}

\subsubsection{Assessment of Redox State}

Redox state was profiling as the total serum antioxidants (TAS) activity and Malondialdehyde (MDA); the TAS activity was evaluated by the phosphomolybdenum method according to the procedure described previously. The assay is based on the reduction of Mo (VI)-Mo (V) by the extract and subsequent formation of a green phosphate/Mo (V) complex $[10,11]$. Also, MDA was monitored by reacting with Thiobarbituric Acid (TBA) to generate the MDA-TBA adduct which can be quantified colorimetrically at $532 \mathrm{~nm}$ [12].

\subsubsection{Angiogenic Markers}

Three angiogenic markers were assessed by quantitative measurement of human VEGF-A in serum used Human VEGF-A ELIZA assay [13], quantitative measurement of serum human ES using Human Endostatin ELIZA [14] and determination of circulating endothelial cells (CECs) in peripheral blood based on isolation of ECEs by Dyna beads. Briefly, $1 \mathrm{ml}$ blood was mixed with $1 \mathrm{ml}$ isolation buffer (Phosphate Buffer Saline (PBS) free from $\mathrm{Ca} 2+$ or $\mathrm{Mg} 2+$, $0.1 \%$ Bovine serum Albumin (BSA), $2 \mathrm{mM}$ EDTA, PH 7.4.) at $4^{\circ} \mathrm{C}$. $20 \mu \mathrm{L}$ blocking agent and $50 \mu \mathrm{L}$ antibody-coated Dynabeads $(10 \mu \mathrm{g} / \mathrm{ml})$ were added and mixed thoroughly. Then the sample was mixed for $30 \mathrm{~min}$ at $4^{\circ} \mathrm{C}$. Between each 
washing procedure, the sample was flushed 4 times with 100 $\mu \mathrm{L}$ buffer. After washing, the cells were suspended in acridine orange $(10 \mathrm{~g} / \mathrm{ml}$ in PBS $)$ and counted with fluorescence microscope (Olympus, USA) [15].

\subsection{Statistical Analysis}

Statistical analysis was performed using SPSS Programmes software package Version 13.0 (SPSS Inc., Chicago, USA). Student t-test was used for the comparison of patients and controls or patients subgroups. Dependent Paired t-test was used to compare the same group or subgroups before mastectomy, after mastectomy, after chemotherapy and after three months later. Differences were considered statistically significant at $\mathrm{p}<0.05$.

\section{Results}

\subsection{Clinical Study}

Clinical and pathological characteristics of the patients included in current study are shown in Table 1 . The age range of the participants in the present study was 35-58 for Gp-I (mean age $=45 \pm 1.7$ ) and 39-67 for Gp-II (mean age $=$ 53.7 \pm 2.1 ). Among Gp-II, $17 \mathrm{BC}$ patients had positive lymph node $(\mathrm{LN})(85 \%)$ and 3 had negative LN (15\%). There were 16 BC patients had positive ER $(80 \%)$ and 4 had negative ER (20\%). There were $15 \mathrm{BC}$ patients had positive PR $(75 \%)$ and 5 had $(25 \%)$ negative PR. It is noticed that there is a decrease of adverse effect of chemotherapy as vomiting, fatigue and nausea in Gp-IIb comparing to Gp-IIa.

Table 1. Characterization of the two subgroups of BC patients, Gp-(IIa) and Gp-(IIb).

\begin{tabular}{|c|c|c|c|c|}
\hline Patients & Age & ER & PR & LN \\
\hline \multicolumn{5}{|c|}{ GP-IIa (BC patients were treated with chemotherapy alone) } \\
\hline 1 & 65 & +++ & +++ & N3 \\
\hline 2 & 39 & + & ++ & N3 \\
\hline 3 & 50 & +++ & +++ & N1 \\
\hline 4 & 54 & + & + & N1 \\
\hline 5 & 43 & ++ & ++ & N3 \\
\hline 6 & 48 & ++ & +++ & N1 \\
\hline 7 & 47 & +++ & +++ & $\mathrm{N} 2$ \\
\hline 8 & 60 & - & - & $\mathrm{N} 2$ \\
\hline 9 & 65 & +++ & +++ & N3 \\
\hline 10 & 45 & + & + & N1 \\
\hline \multicolumn{5}{|c|}{ Gp-IIb (BC patients were treated with chemotherapy plus vitamin D3 and B6) } \\
\hline 11 & 44 & +++ & ++ & N0 \\
\hline 12 & 43 & +++ & +++ & N3 \\
\hline 13 & 67 & + & - & $\mathrm{N} 2$ \\
\hline 14 & 62 & + & + & N0 \\
\hline 15 & 60 & + & + & $\mathrm{N} 2$ \\
\hline 16 & 50 & - & - & N1 \\
\hline 17 & 45 & - & - & N3 \\
\hline 18 & 65 & - & - & N3 \\
\hline 19 & 67 & + & + & N3 \\
\hline 20 & 48 & +++ & ++ & N0 \\
\hline
\end{tabular}

\subsection{Biochemical Investigations}

Comparing to healthy women, highly oxidative stress was detected in BC patients where mean serum MDA 2.07 \pm 0.185 $\mu \mathrm{mol} / 1$ vs TAS $0.39 \pm 0.062 \mu \mathrm{g} / \mathrm{ml}$. It is noticed that persistence of OS throughout the follow up after mastectomy where serum MDA $2.58 \pm 0.282 \mu \mathrm{mol} / 1$ vs TAS $0.32 \pm 0.045 \mu \mathrm{g} / \mathrm{ml}$ ) and persist without insignificant change after chemotherapy alone serum MDA $2.87 \pm 0.231 \mu \mathrm{mol} / \mathrm{l} \mathrm{vs}$ TAS $0.2 \pm 0.039 \mu \mathrm{g} / \mathrm{ml}$ comparing to those supplemented with vitamins serum MDA $1.5 \pm 0.166 \mu \mathrm{mol} / 1$ vs TAS $1.16 \pm 0.089 \mu \mathrm{g} / \mathrm{ml}$. However, BC patients supplemented with vitamins D3 and B6 from the commence of chemotherapy course and continued for three months later show significant decline in OS where serum MDA $0.44 \pm 0.076 \mu \mathrm{mol} / 1$ vs TAS $1.76 \pm 0.121 \mu \mathrm{g} / \mathrm{ml}$ comparing to those supplemented with vitamins for three months only after completion chemotherapy course serum MDA $0.81 \pm 0.175 \mu \mathrm{mol} / 1$ vs TAS $1.27 \pm 0.098 \mu \mathrm{g} / \mathrm{ml}$. Interestingly, it is noticed that at the end of follow up, the level of serum TAS in BC patients supplemented with vitamins D3 and B6 from the commence of chemotherapy and continued for three months later was approximated to the physiological level.

In parallel to OS, significant elevation of VEGF (562 \pm $87.9 \mathrm{pg} / \mathrm{ml}, \quad p$ 0.001) was observed in BC patientsand continued after mastectomy $(313.5 \pm 40.7 \mathrm{pg} / \mathrm{ml}, p<0.001)$ and after chemotherapy alone without insignificant change (177 \pm $8.4 \mathrm{pg} / \mathrm{ml}$ ). However, significant decrease of serum VEGF 
level in BC patients after chemotherapy plus vitamins D3 and B6 $(155 \pm 9.4 \mathrm{pg} / \mathrm{ml}, p<0.05)$. After three months later, significant decrease of serum VEGF level was noticed in BC patients either supplemented with vitamins D3 and B6 from commence of chemotherapy $(144 \pm 10.2 \mathrm{pg} / \mathrm{ml}, \quad p<0.05)$ or those supplemented with vitamins after chemotherapy course $(113.5 \pm 5.9 \mathrm{pg} / \mathrm{ml}, p<0.01)$. On the other hands, serum ES level in $\mathrm{BC}$ patients higher than those in healthy women $(24.6 \pm 2.0 n g / m l v s 22.9 \pm 1.5 n g / m l, p<0.05)$. The higher ES level was continued after mastectomy without significant change $(23.7 \pm 1.9 n g / m l)$, after chemotherapy alone $(28.9 \pm$ $1.2 \mathrm{ng} / \mathrm{ml}, \quad p<0.001)$ or plus vitamins $(38 \pm 1.8 \mathrm{ng} / \mathrm{ml}$, $p<0.001)$. Comparing to its level after chemotherapy, significant increase of serum ES level was noticed in BC patients who supplemented with vitamins from the commence of chemotherapy and continued supplementation for three months later $(48.3 \pm 8.6 \mathrm{ng} / \mathrm{ml}, \mathrm{p}<0.001)$ and those supplemented with vitamins for three months after the ending of chemotherapy course $(38.3 \pm 6.3 \mathrm{ng} / \mathrm{ml}, p<0.01)$. However, no significant difference in serum ES level was noticed between both subgroups at the end of the follow up.

To investigate the impact of oxidative stress on endothelial migration, the present work assesses CECs. Serum CECs in BC patients $(3060 \pm 632$ cell $/ \mathrm{ml})$ was higher than in healthy women $(70 \pm 52 \mathrm{cell} / \mathrm{s} / \mathrm{ml})$. After mastectomy, Serum CECs level was declined $(1775 \pm 443$ cells $/ \mathrm{ml})$ and continued this decline after chemotherapy alone $(960 \pm 383$ cell $/ \mathrm{ml})$, however, this decline was observed clearly in those received chemotherapy plus vitamins D3 and B6 $(600 \pm 163$ cells $/ \mathrm{ml})$. Moreover, serum CECs level in BC patients supplemented with vitamins for three months after chemotherapy course $(400 \pm 210$ cell $\mathrm{s} / \mathrm{ml})$ comparing to those level in BC patients supplemented with vitamins from the commence of chemotherapy and continued for three months (220 \pm 91 cells $/ m l)$.

A

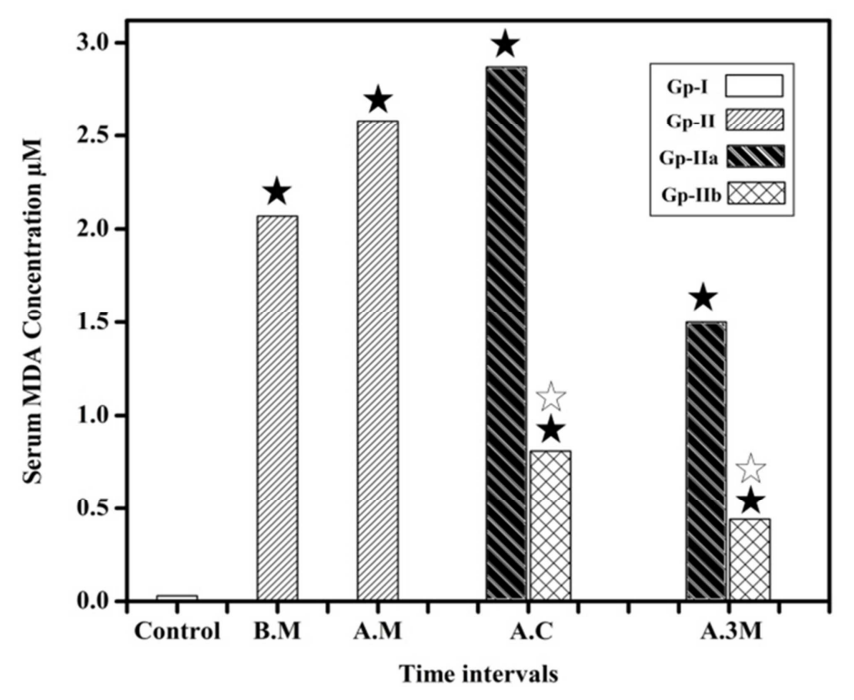

B

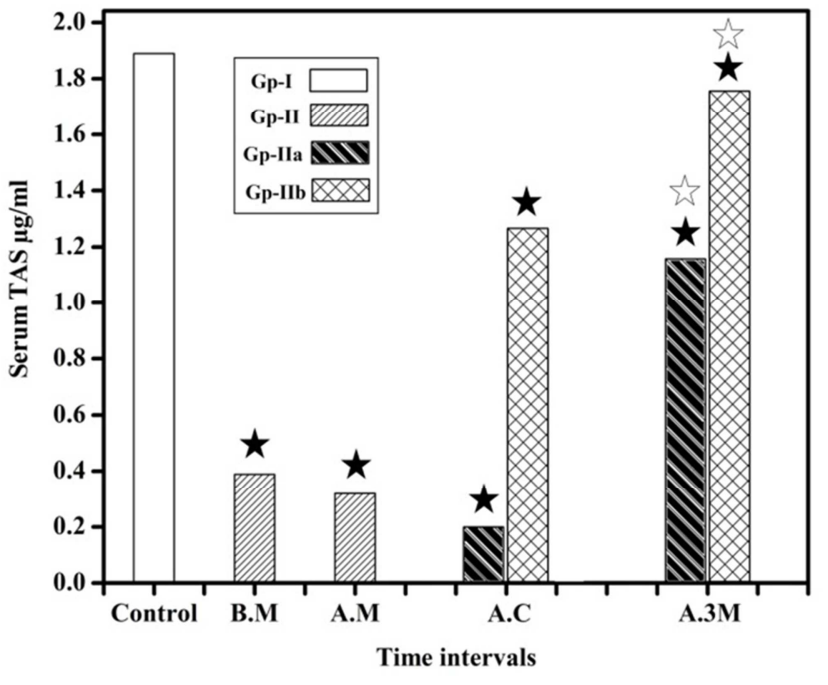

C

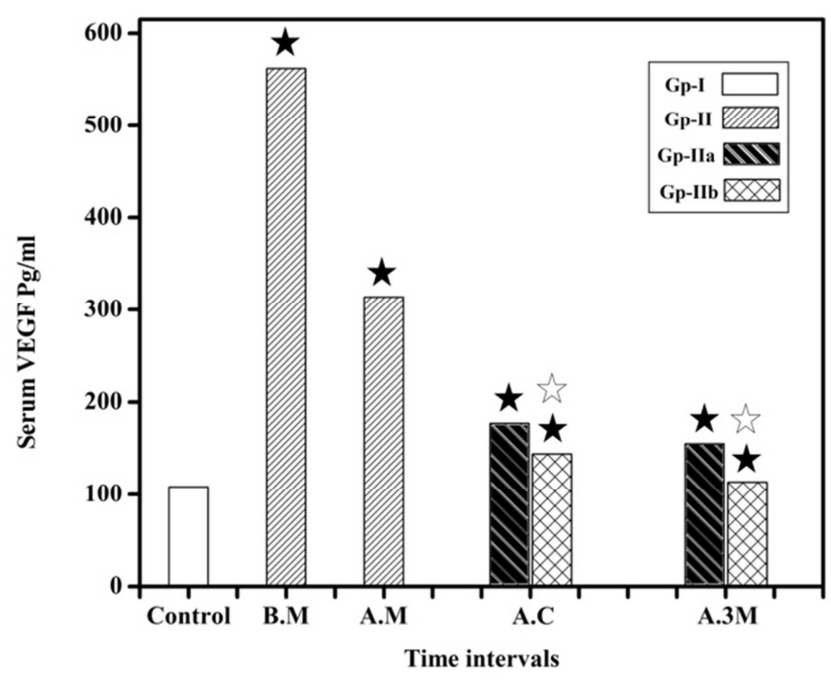

D

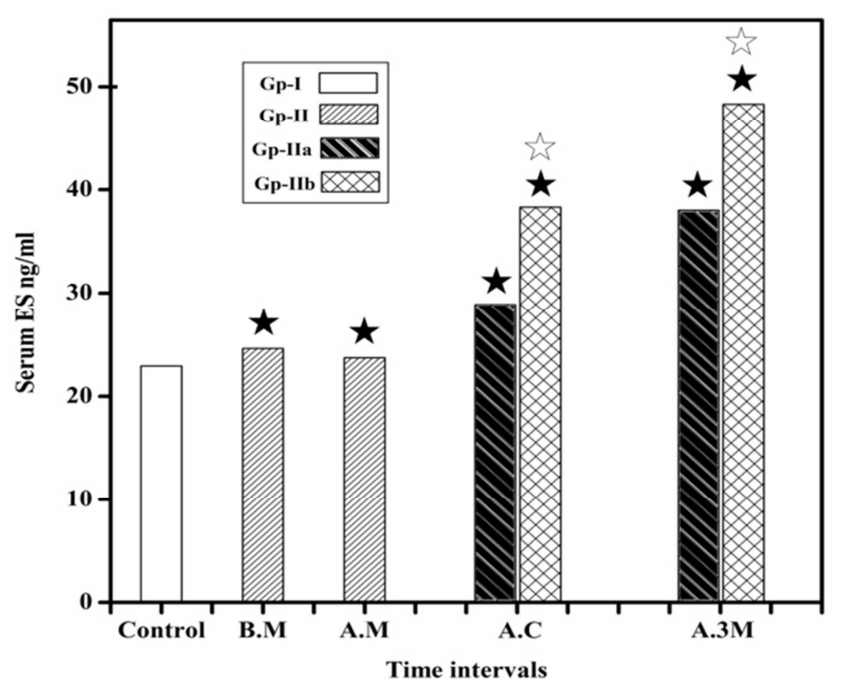


$\mathbf{E}$

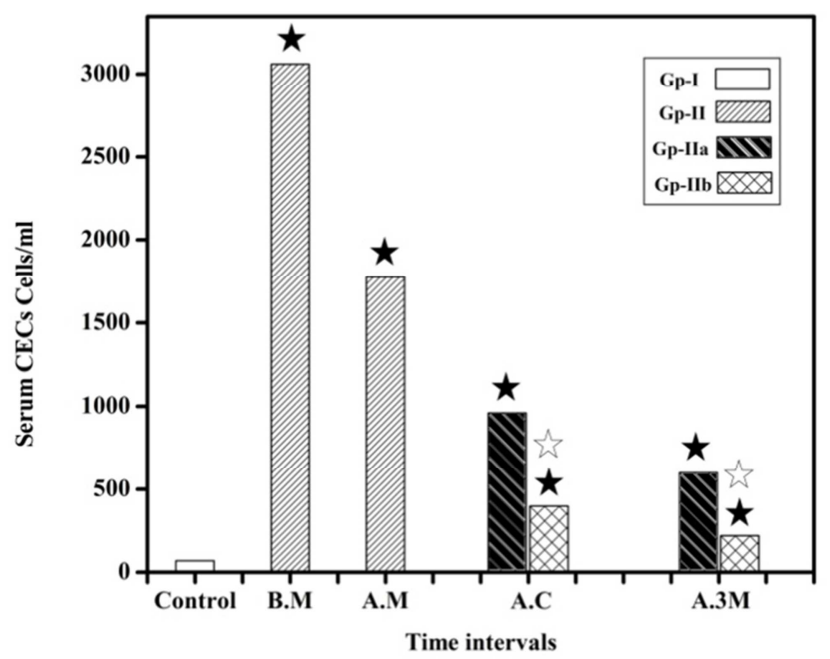

Figure 1. Changes in mean levels of serum MDA in panel A, TAS in panel B, $V E G F$ in panel $C$, Endostatin in panel D, CECs in panel E throughout treatment time intervals in $B C$ patients (Gp-II) and two $B C$ patients subgroups; $B C$ patients were received chemotherapy alone (Gp-IIa) and BC patients were received chemotherapy plus vitamins (Gp-IIb). Black star mentions to significant difference throughout the time intervals. White star mentions to the significant difference between the two subgroups Gp-IIa and $G p-I I b$ at the same time interval. All values are presented as mean $\pm S$. E., significant difference was considered $p<0.05$.
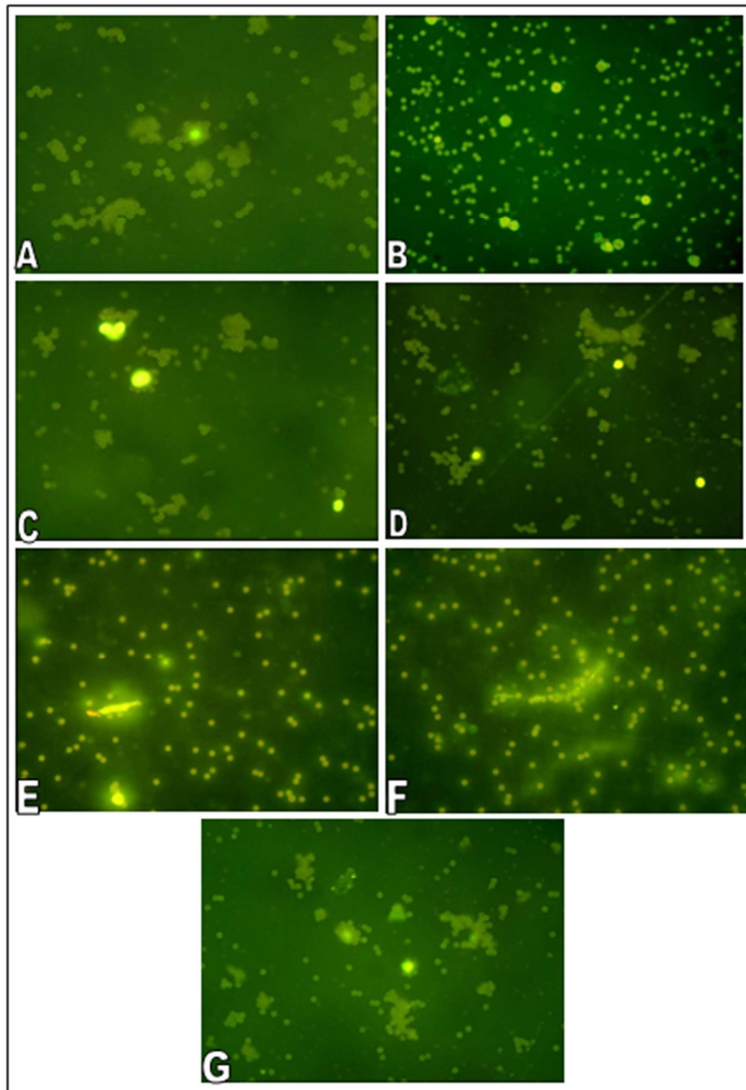

Figure 2. Immunofluorescence analysis of CECs isolated from $G p-(I)$ in panel A, from Gp-(II) B. M. in panel B, Gp-(II) A. M. in panel C, (Gp-IIa) A.C in panel D, (Gp- IIb) A. C in panel E, Gp-(IIa) (A.3M.) in panel F, (GpIIb) (A.3M.) in panel $G$.

\subsection{Correlation Studies}

Serum TAS was negatively correlated with serum VEGF and CECs $(\mathrm{P}<0.001)$ but positively correlated with serum ES $(\mathrm{P}<0.001)$. Serum MDA level was negatively correlated with serum ES $(\mathrm{P}<0.05)$, but positively correlated with serum VEGF and CECs, $(\mathrm{P}<0.01)$ and $(\mathrm{P}<0.001)$, respectively. Serum CECs was negatively correlated with serum ES $(\mathrm{P}<0.001)$ but positively correlated with serum VEGF $(\mathrm{P}<0.001)$ as shown in figure 3 . No correlation was detected between any of the biochemical parameters and clinical parameters (i.e. age, ER, PR and LN) (data not shown).

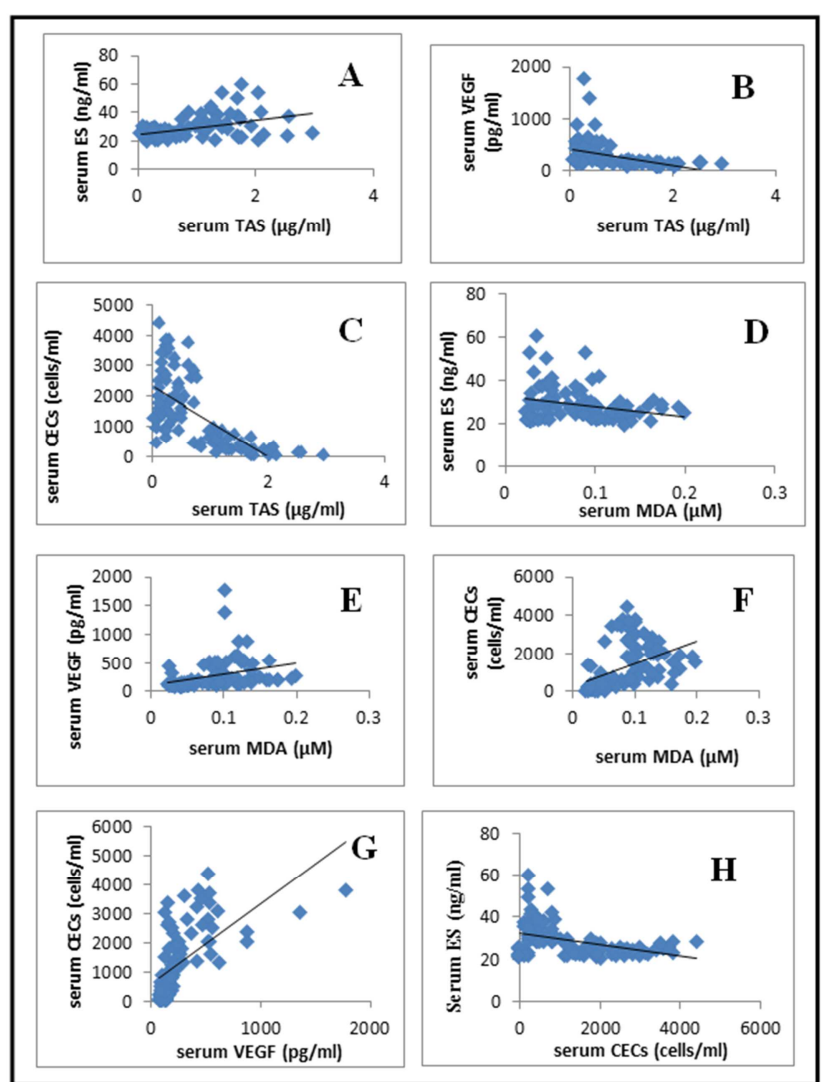

Figure 3. Correlation between serum TAS and serum ES throughout treatment of $B C$ patients, $P<0.001$ in panel $A$, correlation between serum TAS and serum VEGF throughout treatment of $B C$ patients, $P<0.001$ in panel $B$, correlation between serum TAS and serum CECs throughout treatment of $B C$ patients, $P<0.001$ in panel $C$, correlation between serum $M D A$ and serum ES throughout treatment of $B C$ patients, $P<0.05$ in panel $D$, correlation between serum MDA and serum VEGF throughout treatment of $B C$ patients, $P<0.01$ in panel $E$, correlation between serum $M D A$ and serum $C E C$ s throughout treatment of $B C$ patients, $P<0.001$ in panel $F$, correlation between serum VEGF and serum CECs throughout treatment of $B C$ patients, $P<0.001$ in panel $G$, correlation between serum ES and serum CECs throughout treatment of $B C$ patients, $P<0.001$ in panel $H$

\section{Discussion}

In the present study, following mastectomy, most $\mathrm{BC}$ patients underwent treatment with a combination of the anticancer therapy for six cycles including cyclophosphamide, doxorubicin, and 5-fluorouracil (CAF regimen). Administration of supra-dietary doses of 
antioxidant agents during chemotherapy and radiotherapy argues the greatest controversy. A point of view depends on evidence revealed that excessive supplements of antioxidants decrease the efficacy of the chemotherapy and radiotherapy $[16,17]$. On the other hand, several studies have been demonstrated that antioxidants enhance the growth-inhibitory effects of X-irradiation and chemotherapeutic agents on tumor cells, depending upon the dose, form and type of antioxidant, chemotherapeutic agent and tumor [18]. Subsequently, following up the anti-angiogenic effect of supra-dietary doses of vitamins as an adjuvant therapy in BC patients may help in solving the controversy about vitamins supplementation during the conventional anti-cancer therapy and may add a great benefits in ongoing clinical trials studying anti-angiogenic agents, the advanced anti-cancer therapies.

\subsection{Effect of Tumorgenesis}

The observed elevation of OS and CECs in BC patients supports that OS enhances the angiogenesis since MDA disrupts collagen integrity so facilitate ECs migration which have been already proliferate 45 times intratumor faster than ECs in adjacent benign stroma [19]. Although the high level of ES, as a result of lacking elastase in cancer tissues, the active angiogenesis in $\mathrm{BC}$ patients may be explained as high level of VEGF overcome the anti-angiogenic action of ES [20].

\subsection{Effect of Mastectomy}

Numerous cytokines and cells that are involved during the three phases of wound healing were studied. Of these, VEGF, ES and CECs are the major determinants of angiogenic outcome and regulated by redox homeostasis. The persistence of OS accompanied with shifting of angiogenic balance towards anti-angiogenic effect as an observed decline in serum VEGF and CECs levels support theory revealed that the main source of these parameters is tumor and its microenvironment [21]. Consistently, a previous study emerged the role of OS after surgery in VEGF production and ECs migration [22].

\subsection{Effect of Vitamin D3 and B6 Supplementation}

The goal of the present study was to examine the relevance of vitamins D3 and B6 supplementation in combination with chemotherapy, especially, several mechanisms by which chemotherapy induced-OS were documented [23]. In this aspect, several studies hypothesized that high levels of D3 might reduce the risk of $\mathrm{BC}$ through a variety of different mechanisms as inhibition of cellular proliferation, inducing differentiation, apoptosis and inhibition of angiogenesis in normal and malignant breast cell. Also, the anti-carcinogenic effects of D3 on transformed mammary cells have been well characterized and include cell cycle arrest in G0/G1, induction of differentiation markers, and activation of apoptosis. It has been demonstrated that vitamin B6 suppress tumorigenesis, metastasis, anaemia in cancer patients as well as reducing fatigue, irritability, moodiness/depression, fluid retention, and breast tenderness in premenstrual syndrome (PMS), in addition to its antioxidant role. Following mastectomy, BC patients received six cycles of combined chemotherapy. The data showed the persistence of OS in BC patients received chemotherapy alone while significant improving in the antioxidant status was noticed in BCpatients who received chemotherapy plus vitamins D3 and B6. In this aspect, OS resulting from chemotherapeutic agents has a strong consequence in inducing adverse effects including fatigue, nausea and vomiting in addition to enhance angiogenesis progression, metastasis and recurrence in cancer patients $[3,24,25]$. On the other hands, vitamin B6 reduces the OS, inflammation and carcinogenesis, in addition to, reverses side effects in patients receiving chemotherapy without interference with its efficacy and increases survival of cancer patients [26, 27]. Also, it was revealed that supplementation with vitamin D3 contributed to diminish MDA level [28].

Despite the insignificant change of ES level, the observed decline in CECs emphasis the anti-angiogenic action of vitamin D3 and B6 in combination with chemotherapy comparing to those received chemotherapy alone. The insignificant change in serum ES level between the two subgroups doesn't mean that vitamins D3 and B6 supplementation have no effect on ES action since it has been demonstrated that the anti-angiogenic and antitumor activity of ES operates over a biphasic curve meaning that higher serum levels are less active than lower levels [29]. This means that vitamins D3 and B6 supplementation may help in maintaining ES within its active level. In this aspect, it has been demonstrated that the anti-proliferative effect of vitamins D3 and vitamin B6 contributed to improve endothelial function and systemic inflammation $[30,31]$. However, controversial data exist in the literature regarding to the effect of vitamin D3 on VEGF expression while a study revealed that vitamin D3 suppresses VEGF expression another study showed that vitamin D3 increases VEGF expression. One explanation is that vitamin B6 regress the activation action of vitamin D3 on VEGF pathway. Another explanation may be that the action of vitamin D3 in normal tissues differs from that in tumor tissues [32- 34]. Collectively, the present study reveals that the anti-angiogenic action of vitamin D3 and B6 isn't limited to reduce angiogenesis via decreasing VEGF level but also keep ES within its active level suggesting that the combination of vitamins D3 and B6 strengthen the anti-angiogenic action of chemotherapy and thus interfere with metastasis in BC patients. Thus, the present findings may add benefits in selecting treatment regimen that based on combination of anti-oxidants and anti-angiogenic therapy since many ongoing clinical trials based on anti-angiogenic therapy [35].

Another goal of the present study was to study whether vitamins D3 and B6 supplementation with chemotherapy or after chemotherapy course is preferable. So it was designed to supplement both subgroups of $\mathrm{BC}$ patients with vitamin 
D3 and B6 for three months later either who were received chemotherapy alone or chemotherapy plus vitamins. Especially, after completion of chemotherapy cycles, most BC patients always treated with radiotherapy which enhanced generation of superoxide agents resulting in deficiency in micronutrients. This deficiency may be more detrimental than radiation itself. It has been demonstrated that micronutrients may selectively induce apoptosis, inhibit cell proliferation in cancer cells in addition to spare normal cells [36]. In this aspect, the present data showed that BC patients received vitamins D3 and B6 from the beginning of chemotherapy course and continued vitamins supplementation for three months later during radiotherapy course show significant decline in CECs compared to BC patients received vitamins D3 and B6 for three months after completion of chemotherapy course. However, there is no data about enumeration of CECs after radiotherapy course. Also, our findings underscore the impact of vitamins D3 and B6 in reducing OS and strengthen the anti-angiogenic effect of radiotherapy. This aspect gains support by observations that vitamin D3 enhances the cytotoxic effects of gamma irradiation. In addition; vitamin B6 counteracts the side effects of radiotherapy and enhances the survival rate of cancer patients [37-39]. Our data emerge the potential interaction between vitamin B6 and D3 in improving the clinical outcomes of cancer patients and development of new therapeutic approaches to prevent invasion and metastasis of tumors locally relapsing after radiotherapy.

To our knowledge, it is the first time reports the role of vitamins D3 and B6 in decreasing the level of CECs in serum meaning that their effectiveness in counteracting the cancer progression and reduce the side effect of chemotherapy and radiotherapy.

The small sample size is the limitation of the present study.

\section{Conclusion}

It can be concluded that vitamins D3 and B6 strengthen the anti-angiogenic action of chemo- and radiotherapy and subsequently interfering with metastasis, in addition to minimize the side effects of chemo- and radiotherapy as nausea, fatigue and vomiting and improving the clinical outcomes as well as the efficacy of the conventional anticancer therapies. In this aspect, the multiple actions of vitamins may be extended beyond their anti-oxidation to antiangiogenesis and interfering with cancer progression. From this view, wide ranges of studies are required to formulate the proper schedule based on combination between vitamins, chemotherapy and radiotherapy.

\section{Competing Interests}

The authors declare that there is no competing interest.

\section{Author Contribution}

HF carried outthe biochemical investigations and prepared the draft of manuscript. MA participated in analysis and interpretation of data analysis, revised the article of the research. KS participated in the surgical part of research. HK supervised the clinical investigations of the research. SE designed the research, supervised the biochemical investigations of the research. All authors read and approved the final manuscript.

\section{Acknowledgements}

I would like to express my thanks and deepest appreciation to Prof. Dr. Lobna A. M. Shamaa, Immunology department, Medical Research Institute, Alexandria University, for her honest assistance in microscopic examination of CECs by Fluroscence microscope. I am particularly grateful for Dr. Mohamed S. Abdel-Latif. Lecturer of microbiology, Medical Laboratory department, Pharos University, for critically revising the final draft for manuscript.

\section{Funding}

This research did not receive any specific grant from funding agencies in the public, commercial, or not-for-profit sectors.

\section{Abbreviations}

$\begin{array}{ll}\text { BC } & \text { Breast cancer } \\ \text { BRCA-1 } & \text { Breast cancer associated gene-1 } \\ \text { CECs } & \text { Circulating endothelial cells } \\ \text { ES } & \text { Endostatin } \\ \text { ECs } & \text { Endothelial cells } \\ \text { ER } & \text { Estrogen receptor } \\ 5 \text {-FU } & \text { 5-fluorouracil } \\ \text { MDA } & \text { Malondialdehyde } \\ \text { OS } & \text { Oxidative stress } \\ \text { PR } & \text { Progestogen receptor } \\ \text { ROS } & \text { Reactive oxygen species } \\ \text { TAS } & \text { Total antioxidant status } \\ \text { VEGF } & \text { Vascular endothelial growth factor }\end{array}$

\section{References}

[1] Dey S, Soliman, AS, Hablas A, Seifeldein IA, Ismail K, Ramadan M, El-Hamzawy H, Wilson ML, Banerjee M, Boffetta P, Harford J, Merajver SD: Urban-rural differences in breast cancer incidence in Egypt (1999-2006). Breast 2010; 19(5): 417-23.

[2] McPherson K, Steel CM, Dixon JM: Breast cancer, epidemiology, risk factors, and genetics. BMJ 2000; 321: 624-628.

[3] Brown NS and Bicknell R: Hypoxia and oxidative stress in breast cancer Oxidative stress: its effects on the growth, metastatic potential and response to therapy of breast cancer. Breast Cancer Res 2001; 3: 323-327.

[4] Kim YW and Byzova TV: Oxidative stress in angiogenesis and vascular disease. Blood 2014; 123 (5): 625-31. 
[5] Goon PKY, Lip GYH, Boos CJ, Stonelake PS, and Blann AD. Circulating Endothelial Cells, Endothelial Progenitor Cells, and Endothelial Microparticles in Cancer. Neoplasia 2006; 8(2): 79-88.

[6] Bidard FC, Mathiot C, Degeorges A, Etienne-Grimaldi MC, Delva R, Pivot X, Veyret C, Bergougnoux L, de Cremoux P, Milano G and Pierga JY: Clinical value of circulating endothelial cells and circulating tumor cells in metastatic breast cancer patients treated first line with bevacizumab and chemotherapy. Ann Oncol 2010; 21: 1765-71.

[7] Campbell MJ, Gombart AF, Kwok SH, Park S, Koeffler HP: The anti-proliferative effects of 1, $25(\mathrm{OH}) 2 \mathrm{D} 3$ on breast and prostate cancer cells are associated with induction of BRCA1 gene expression. Oncogene 2000; 19: 5091-7.

[8] Cheng $\mathrm{CH}$, Chang SJ, Lee BJ, Lin KL and Huang YC: Vitamin B6 supplementation increases immune responses in critically ill patients. Eur. J Clin. Nutr 2006; 60:1207-13.

[9] Zhang XH, Ma J, Smith-Warner SA, Lee JE, Giovannucci E. Vitamin B6 and colorectal cancer: current evidence and future directions. World J Gastroenterol 2013; 19(7):1005-10.

[10] Prieto P, Pineda M and Aguilar M: Spectrophotometric quantitation of antioxidant capacity through the formation of a Phosphomolybdenum Complex: Specific application to the determination of vitamin E. Analytical Biochemistry 1999; 269: 337-341.

[11] Pendyala G, Thomas B and Kumari S: The challenge of antioxidants to free radicals in periodontitis. J Indian Soc. Periodontol 2008; 12: 79-83.

[12] Tug T, Karatas F, Terzi SM, Ozdemir N. Comparison of Serum Malondialdehyde Levels Determined by Two Different Methods in Patients with COPD: HPLC or TBARS Methods. Laboratory Medicine 2005; 36: 41-44.

[13] Byrne GJ, Mcdowell G, Agarawal R, Sinha G, Kumar S and Bundred NJ. Serum Vascular Endothelial Growth Factor in Breast Cancer. Anticancer Research 2007; 27: 3481-3488.

[14] Sasaki T, Larsson H, Tisi D, Claesson-Welsh L, Hohenester E and Timpl R. Endostatins derived from collagens XV and XVIII differ in structural and binding properties, tissue distribution and anti-angiogenic activity. J Mol. Biol. 2000; 301: 1179-1190.

[15] Woywodt A, Blann AD, Kirsch T, Erdbruegger U, Banzet N, Haubitz M. Isolation and enumeration of circulating endothelial cells by immunomagnetic isolation: proposal of a definition and a consensus protocol. J Thromb. Haemost 2006; 4(3): 671-7.

[16] D'Andrea GM. Use of Antioxidants During Chemotherapy and Radiotherapy Should Be Avoided. CA Cancer J Clin 2005; 55: 319-321.

[17] Moss RW: Do Antioxidants Interfere With Radiation Therapy for Cancer? Integr Cancer Ther 2007; 6: 281-292.

[18] Weijl NI, Elsendoorn TJ, Lentjes EG, Hopman GD, WipkinkBakker A, Zwinderman AH, Cleton FJ, Osanto S: Supplementation with antioxidant micronutrients and chemotherapy-induced toxicity in cancer patients treated with cisplatin-based chemotherapy: a randomised, double-blind, placebo-controlled study. Eur J Cancer 2004; 40:1713-23.
[19] Slatter DA, Avery NC, Bailey AJ:Identification of a new cross-link and unique histidine adduct from bovine serum albumin incubated with malondialdehyde. J Biol. Chem 2004; 279: 61-9.

[20] Zhao J, Yan F, Ju H, Tang J and Qin J. Correlation between serum vascular endothelial growth factor and endostatin levels in patients with breast cancer. Cancer Letters 2004; 204: 87-95.

[21] Wu FP, Hoekman K, Meijer S, Cuesta MA: VEGF and endostatin levels in wound fluid and plasma after breast surgery. Angiogenesis 2003; 6(4): 255-7.

[22] Conklin KA: Chemotherapy-Associated Oxidative Stress: Impact on Chemotherapeutic Effectiveness. Integrative Cancer Therapies 2004; 3: 294-300.

[23] Nicolson GL. Lipid Replacement Therapy: A Nutraceutical Approach for Reducing Cancer-Associated Fatigue and the Adverse Effects of Cancer Therapy while Restoring Mitochondrial Function. Cancer and Metastasis Reviews 2010; 29(3): 543-552.

[24] Areti A, Yerra VG, Naidu VGM and Kumar A: Oxidative stress and nerve damage: Role in chemotherapy induced peripheral neuropathy. Redox Biol 2014; 2: 289-295.

[25] Shen J, Lai CQ, Mattei J, Ordovas JM, Tucker KL. Association of vitamin B-6 status with inflammation, oxidative stress, and chronic inflammatory conditions: the Boston Puerto Rican Health Study. Am J Clin. Nutr 2010; 91: 337-342.

[26] Simone CB II, Simone NL, Simone V and Simone CB: Antioxidants and other nutrients do not interfere with chemotherapy or radiotherapy and can increase kill and increase survival part. Alternative therapies 2007; 13: 22-28.

[27] Suwannalert P, Rattanachitthawat S, Chaiyasut C, and Riengrojpitak S: High levels of 25-hydroxyvitamin D3 [25(OH)D3] and $\alpha$-tocopherol prevent oxidative stress in rats that consume Thai brown rice. Journal of Medicinal Plants Research 2010; 4(2):120-124.

[28] Celik I, Surucu O, Dietz C, Heymach JV, Force J, Ho“scheleI, Becker CM, Folkman J and Kisker O: Therapeutic Efficacy of Endostatin Exhibits a Biphasic Dose-Response Curve. Cancer Res2005; 65: 11044- 11050.

[29] ChungI, Han G, Seshadri M. GillardBM, Yu WD, Barbara A. Foster BA, Trump DL and Johnson CS. Role of Vitamin D Receptor in the Antiproliferative Effects of Calcitriol in Tumor-Derived Endothelial Cells and Tumor Angiogenesis In vivo. Cancer Res 2009; 69(3): 967-975.

[30] Rossi D and Catalano G: Pyridoxine as prophylactic therapy for palmar-plantar erythrodysesthesia associated with administration of pegylated liposomal doxorubicin (caelyx): a single-center experience. Oncology 2007; 73: 277-8.

[31] Ben-Shoshan M, Amir S, Dang DT, Dang, LH, Weisman Y, Mabjeesh NJ: 1alpha, 25-dihydroxyvitamin D3 (Calcitriol) inhibits hypoxia-inducible factor-1/vascular endothelial growth factor pathway in human cancer cells. Mol. Cancer Ther $2007 ; 6: 1433-9$.

[32] Grundmann $M$, Haidar $M$, Placzko S, Niendorf $R$, Darashchonak N, Hubel CA, von Versen-Höynck F: Vitamin $\mathrm{D}$ improves the angiogenic properties of endothelial progenitor cells. American Journal of Physiology - Cell Physiology2012; 303: 954-962. 
[33] Umeda T, Abe H, Cho H, Shimizu T, Mori T, Kubota Y, Kawai Y, Tanaka M, Kurumi Y, Tani T: An effective case of liver metastasis of breast cancer treated with capecitabine + docetaxel combination therapy using vitamin B6. GanTo Kagaku Ryoho 2010; 37: 687-9.

[34] Lopes N, Paredes J, Costa JL, Ylstra B, Schmitt F. Vitamin D and the mammary gland: a review on its role in normal development and breast cancer. Breast Cancer Research 2012; 14(3):211-217.

[35] Rugo HS. Bevacizumab in the Treatment of Breast Cancer: Rationale and Current Data. The Oncologist 2004; 9: 43-49.

[36] Gröber U, Holzhauer P, Kisters K. Holick MF and Adamietz IA. Micronutrients in Oncological Intervention. Nutrients. 2016; 8(3): 163.
[37] Ma Y, Trump DL and Johnson CS: Vitamin D in combination cancer treatment. J Cancer 2010; 1: 101-107.

[38] Je Y, Lee JE, Ma J, Zhang X, Cho E, Rosner B, Selhub J, Fuchs CS, Meyerhardt J, and Giovannucci E:Prediagnostic plasma vitamin B6 (pyridoxal 5'-phosphate) and survival in patients with colorectal cancer. Cancer Causes Control 2013; 24(4):719-29.

[39] Kuonen F, Secondini C, Rüegg C. Molecular pathways: emerging pathways mediating growth, invasion, and metastasis of tumors progressing in an irradiated microenvironment. Clinical Cancer Research 2012; 18(19): 5196-202. 